\title{
RECENT INCREASE IN COLISTIN-RESISTANT EXTENSIVELY DRUG RESISTANT ACINETOBACTER INFECTIONS AT A TERTIARY CARE CENTER IN PAKISTAN
}

Nosheen Nasir ( $\nabla$ nosheen.nasir@aku.edu )

Aga Khan University https://orcid.org/0000-0003-1610-8748

Fatima Sharif

Aga Khan University

Rubab Mansoor

Aga Khan University

Shehryar Ahmed

Aga Khan University

Bushra Jamil

Aga Khan University

Faisal Mahmood

Aga Khan University

Research article

Keywords: Acinetobacter, Multi-drug resistance, Colistin

Posted Date: July 20th, 2019

DOI: https://doi.org/10.21203/rs.2.11662/v1

License: () (i) This work is licensed under a Creative Commons Attribution 4.0 International License. Read Full License 


\section{Abstract}

Background Acinetobacter is an important nosocomial pathogen and a major cause of morbidity and mortality in hospitalized patients. Recently, colistin resistant strains of Acinetobacter were reported from different parts of the world. We are describing a case series of 18 patients with colistin resistant Acinetobacter over a span of 4 years. Methods Patients with any clinical specimen positive for colistin resistant Acinetobacter from 2014 to 2017 were identified from the hospital records. Three cases were isolated between 2014 and 2015, six cases in 2016 and 9 cases in 2017. Data on patients' demographics as well as clinical data was collected retrospectively on a structured proforma from the hospital medical records. Results Mean age of the patients was $50 \pm 18$ years. Fifteen (83.3\%) out of the 18 patients were male. Acinetobacter Pneumonia was the most common diagnosis in $n=13(72.2 \%$ of the patients). Nine $(50 \%)$ of the patients developed sepsis. In addition to Colistin resistance, carbapenem and amikacin resistance was documented to be $94 \%$ and $61 \%$ respectively. Colistin and carbapenem based combinations were used to treat all patients with a mean antibiotic duration of $20 \pm 10$ days. Median length of hospital stay was 25 days (range 8 - 61), with 14 patients (77.8\%) requiring ICU admission. Eight (44.4\%) of the patients expired and only $6(33.3 \%)$ achieved microbiological eradication. Conclusion Infections due to Colistin resistant strains of Acinetobacter are rapidly increasing, have limited antimicrobial treatment options and are associated with poor outcomes.

\section{Background}

Acinetobacter is an important nosocomial pathogen and a major cause of morbidity and mortality in hospitalized patients (1). It causes hospital-acquired pneumonia, central line associated bloodstream infections as well as surgical site infections (2). Infections caused by Acinetobacter $s p$. are especially difficult to treat due to increasing carbapenem resistance and surveillance studies have shown that the percentage of carbapenem-resistant isolates have gradually increased over the last ten years in Europe, North America, South Asia and Latin America (3). Hence it was included among the six ESKAPE organisms responsible for causing difficult to treat nosocomial infections by the Infectious Diseases Society of America (4).

Increasingly worrisome are reports of emergence of colistin resistant Acinetobacter species. Colistin is usually considered an antibiotic of last resort against multidrug resistant gram negative organisms (5). However, in a surveillance study from US, colistin resistance was reported in approximately $5 \%$ of all Acinetobacter strains (6). This has been attributed to prior colistin use (7). However data is still sparse regarding other predisposing factors. There are also no standard guidelines for treatment but several studies recommend combination therapies such as colistin in combination with carbapenem or sulbactam in multi-drug resistant, carbapenem resistant Acinetobacter infections $(8,9)$. It is still unclear as to which treatment regimen is optimal for use in patients infected with colistin resistant Acinetobacter species as well as the clinical course and prognosis of these patients. Therefore, in this study, we aim to share our experience of managing infections due to colistin resistant Acinetobacter species over a period of 4 years at a tertiary care hospital in Pakistan. 


\section{Methods}

Using microbiological records between 2014 and 2017, patients with any clinical specimen positive for colistin resistant Acinetobacter were identified at the Aga Khan University Hospital Karachi (a 700 bedded tertiary care center). Identification and susceptibility of Acinetobacter species isolated from cultures were performed by automated systems in accordance with Clinical Laboratory Standards Institute (CLSI) recommendations. Moreover, these isolates were susceptible to only one or two antimicrobial categories i.e extensively drug-resistant (XDR) (10). Data on demographics and clinical data were collected a using a structured proforma from the hospital medical records. Variables included age, gender, admitting diagnosis, co-morbids, exposure to invasive devices, site of infection. The Charlson comorbidity index and the qSOFA score were calculated. The definitions of the types of infection was in accordance with Centers for Disease Control and Prevention/National Healthcare Safety Network (11). The outcomes assessed included inhospital mortality, readmission at 30 days, clinical recovery and microbiological outcome. Clinical recovery was defined as the resolution of symptoms at the time of discharge, regardless of microbiological outcome. Patients were categorized as having not recovered if they died during hospital stay or left against medical advice while clinically deteriorating. Microbiological outcome was defined according to the results of the final bacterial culture at the time of discharge. "Eradication" was defined as no growth in the final culture; "persistence" if the pathogen was isolated despite antibiotic treatment and "undetermined" if no culture was done within 24 hours of discharge/mortality.

The study was submitted for ethical approval to the Aga Khan University hospital's ethical review committee and received exemption (5286-Med-ERC-18). Data was anonymized and no personal identifiers were recorded.

\section{Results}

Three cases were identified between 2014 and 2015, six cases in 2016 and 9 cases in 2017. The clinical characteristics of the patients are summarized in table 1. Mean age of the patients was $50 \pm 18$ years. Fifteen (83.3\%) of the 18 patients were male. Fifty percent of the patients had a Charlson comorbidity index of $\geq 3$. Sixty seven $(n=12)$ patients had a qSOFA score of greater than or equal to 2 . Pneumonia was the most frequent diagnosis in $13(72.2 \%)$ of the patients. Only 1 patient had bacteremia which was due to a ventilator- associated pneumonia while 9 (50\%) patients developed sepsis.

In addition to colistin resistance, carbapenem and amikacin resistance was documented to be $94 \%$ and $61 \%$ respectively. All isolates were non-susceptible to piperacillin tazobactam, $83 \%$ were non-susceptible to trimethoprim-sulfamethoxazole. Out of nine specimens which were checked for minocycline susceptibility, only one was non-susceptible. Tigecycline susceptibility was available for six cases and none were sensitive. One of the isolates was resistant to all drugs except minocycline. Drug susceptibilities are shown in Figure 1.

Figure 1: Antimicrobial susceptibility pattern of Acinetobacter species 
Colistin with carbapenem based combinations were used to treat all patients except one with a mean antibiotic duration of $20 \pm 10$ days. Half the patients $(n=9)$ received treatment with an antibiotic to which the colistin resistant Acinetobacter was susceptible. The drugs commonly used in combination included minocycline in 3 patients, tigecycline in 3 patients, aminoglycosides in 3 patients (tobramycin or amikacin) and cefoperazone-sulbactam in 4 patients although sensitivity to sulbactam was not available.

The median length of hospital stay was 25 days (range 8 - 61), with 14 patients (77.8\%) having ICU stay. Eight (44.4\%) of the patients died during their hospital stay (Table 2). Microbiological outcome could be assessed for nine cases including six survivors and three who died. Six (66\%) patients out of 9 achieved microbiological eradication including 3 who died and 3 who survived.

\section{Discussion}

Carbapenem resistant, colistin resistant Acinetobacter is emerging as a major pathogen causing nosocomial infections. There is a dearth of literature describing clinical characteristics of patients and our case series is the first from Pakistan. We have identified 15 cases over a span of only 2 years highlighting the fact that colistin resistant Acinetobacter infections are increasing at an alarming rate.

The treatment options for this organism are limited and include combination therapy with 3 to 4 drug regimen (8). We observed a higher mortality rate of $44 \%$ during hospital stay as compared to 30 -day mortality rate of $30 \%$ in the case series from US (7). Two thirds of our cases required ICU stay compared to $62 \%$ in a study from Greece (12). Pneumonia was seen in $72 \%$ of our cases which was similar to the US study (7) though the study from Greece reported pneumonia in $42 \%$ with bloodstream infections being the most common in their center (12). There was no difference in mortality despite including a drug to which the organism was sensitive on culture as compared to other case series where combination treatment with carbapenem, colistimethate sodium and ampicillin sulbactam was associated with better outcome (7). The antimicrobial susceptibility was similar to the case series reported from US except for amikacin which was non-susceptible in $61 \%$ in our study compared to $94 \%$ (7). In our study, 8 out of 9 specimens in which minocycline susceptibility was checked were found to be sensitive to this drug. Previous studies do indicate a potential role of minocycline in combination with colistin for MDR Acinetobacter, however data on the use of this combination is limited (13).

To the best of our knowledge, this is the first case series from South Asia describing clinical characteristics of patients with colistin resistant Acinetobacter infections. However, we do not have molecular typing for our isolates although we do have data from our center where isolates were all positive for $\mathrm{Bla}_{\text {OxA-51-like, }}$

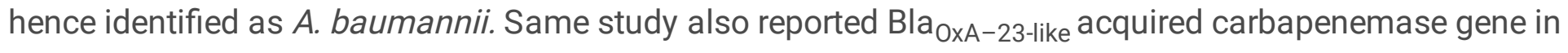
the majority of Carbapenem resistant strains of Acinetobacter (14). Our study has limited generalizability as it is from a single center. However, it highlights the urgency and importance of data from this region.

\section{CONCLUSION}


Infections due to Colistin resistant strains of Acinetobacter are rapidly increasing, have limited antimicrobial treatment options and are associated with poor outcomes.

\section{List Of Abbreviations}

MDRO: Multi-drug resistant organisms

XDR: Extensively drug-resistant

ESKAPE: Acronym for six pathogens: Enterococcus faecium, Staphylococcus aureus, Klebsiella pneumonia, Acinetobacter baumannii, Pseudomonas aeruginosa, Enterobacter spp.

ICU: Intensive care unit

CLSI: Clinical Laboratory Standards Institute

qSOFA: quick Sepsis Related Organ Failure Assessment

ERC: Ethics review committee

\section{Declarations}

\section{Ethics approval and consent to participate}

The study proposal was submitted for ethical approval to the Aga Khan University Ethical Review Committee (ERC) which granted approval of the submission for exemption (5286-Med-ERC-18). Approval by ERC implies administrative permission to proceed with the study including accessing raw data. Data was anonymized and no personal identifiers were recorded in the data set.

\section{Consent for publication}

Not applicable

\section{Availability of data and material}

All data generated or analysed during this study are included in this published article.

\section{Competing interests}

The authors declare that they have no competing interests 


\section{Funding}

None

\section{Authors' contributions}

NN conceived idea, supervised data collection, major contributor of manuscript

FS performed data collection and contribution in writing manuscript

SA performed Data collection and contributed to manuscript

RM perfomed Data collection and contributed to manuscript

BJ reviewed manuscript and expert opinion and supervised the study

FM conceived idea, reviewed manuscript, provided expert opinion and supervised the study

All authors discussed the results and contributed to the final manuscript

\section{Acknowledgements}

None

\section{Author Information}

Not applicable

\section{References}

1.Munoz-Price LS, Weinstein RA. Acinetobacter infection. N Engl J Med. 2008;358(12):1271-81.

2.Peleg AY, Seifert H, Paterson DL. Acinetobacter baumannii: emergence of a successful pathogen. Clin Microbiol Rev. 2008;21(3):538-82.

3.Gilad J, Carmeli Y. Treatment options for multidrug-resistant Acinetobacter species. Drugs. 2008;68(2):165-89.

4.Boucher HW, Talbot GH, Bradley JS, Edwards JE, Gilbert D, Rice LB, et al. Bad bugs, no drugs: no ESKAPE! An update from the Infectious Diseases Society of America. Clinical infectious diseases: an official publication of the Infectious Diseases Society of America. 2009;48(1):1-12. 
5.Mendelson M, Brink A, Gouws J, Mbelle N, Naidoo V, Pople T, et al. The One Health stewardship of colistin as an antibiotic of last resort for human health in South Africa. The Lancet Infectious diseases.

2018;18(9):e288-e94.

6.Queenan AM, Pillar CM, Deane J, Sahm DF, Lynch AS, Flamm RK, et al. Multidrug resistance among Acinetobacter spp. in the USA and activity profile of key agents: results from CAPITAL Surveillance 2010. Diagnostic microbiology and infectious disease. 2012;73(3):267-70.

7.Qureshi ZA, Hittle LE, O'Hara JA, Rivera JI, Syed A, Shields RK, et al. Colistin-resistant Acinetobacter baumannii: beyond carbapenem resistance. Clinical infectious diseases: an official publication of the Infectious Diseases Society of America. 2015;60(9):1295-303.

8.Batirel A, Balkan II, Karabay O, Agalar C, Akalin S, Alici O, et al. Comparison of colistin-carbapenem, colistin-sulbactam, and colistin plus other antibacterial agents for the treatment of extremely drug-resistant Acinetobacter baumannii bloodstream infections. Eur J Clin Microbiol. 2014;33(8):1311-22.

9.Zalts R, Neuberger A, Hussein K, Raz-Pasteur A, Geffen Y, Mashiach T, et al. Treatment of CarbapenemResistant Acinetobacter baumannii Ventilator-Associated Pneumonia: Retrospective Comparison Between Intravenous Colistin and Intravenous Ampicillin-Sulbactam. Am J Ther. 2016;23(1):E78-E85.

10.Magiorakos AP, Srinivasan A, Carey RB, Carmeli Y, Falagas ME, Giske CG, et al. Multidrug-resistant, extensively drug-resistant and pandrug-resistant bacteria: an international expert proposal for interim standard definitions for acquired resistance. Clinical microbiology and infection: the official publication of the European Society of Clinical Microbiology and Infectious Diseases. 2012;18(3):268-81.

11. Horan TC, Andrus M, Dudeck MA. CDC/NHSN surveillance definition of health care-associated infection and criteria for specific types of infections in the acute care setting. American journal of infection control. 2008;36(5):309-32.

12.Oikonomou O, Sarrou S, Papagiannitsis CC, Georgiadou S, Mantzarlis K, Zakynthinos E, et al. Rapid dissemination of colistin and carbapenem resistant Acinetobacter baumannii in Central Greece: mechanisms of resistance, molecular identification and epidemiological data. BMC infectious diseases. 2015;15:559.

13.Greig SL, Scott LJ. Intravenous Minocycline: A Review in Acinetobacter Infections. Drugs. 2016;76(15):1467-76.

14.Irfan S, Turton JF, Mehraj J, Siddiqui SZ, Haider S, Zafar A, et al. Molecular and epidemiological characterisation of clinical isolates of carbapenem-resistant Acinetobacter baumannii from public and private sector intensive care units in Karachi, Pakistan. J Hosp Infect. 2011;78(2):143-8.

\section{Tables}

Table 1: Characteristics and Outcomes of Patients with Colistin-Resistant Acinetobacter 
Page $8 / 12$ 
Patient Gender Age Charlson qSOFA Site Of Antibiotic Sensitive Length ICU In Microbiological group Comorbidity Score Infection Regimen Antibiotic Of Admission Hospital Outcome Index

Used Stay In Mortality

Days

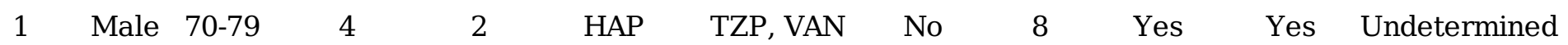

2 Male $60-69 \quad 4 \quad 2 \quad$ HAP $\quad$ MEM, $\quad$ Yes $16 \quad$ No $\quad$ Yes Eradication

CST, MIN

3 Male $70-79 \quad 4 \quad 3 \quad$ VAP $\quad$ MEM, $\quad$ Yes $29 \quad$ No $\quad$ No Eradication CST, TOB,

\section{CES}

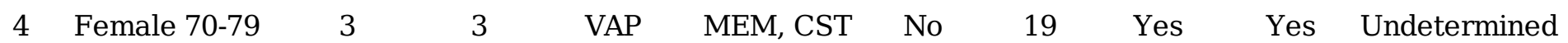

5 Female 40-49 $5 \quad 2 \quad$ VAP $\quad$ MEM, $\quad$ Yes 42 Yes $\quad$ Yes Eradication CST, SXT

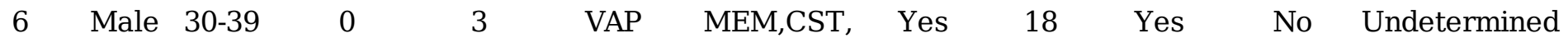
MIN, RIF

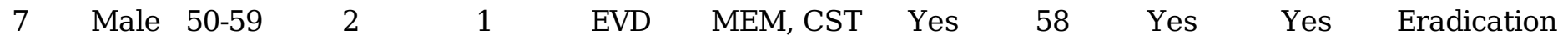
Ventriculitis (IV \&IT),

AMK (IV

\& IT),

MIN

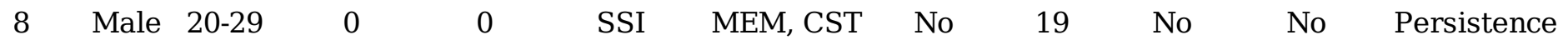

9 Male $40-49 \quad 0 \quad 3 \quad$ HAP $\quad$ MEM, CST $\quad$ No $13 \quad$ Yes $\quad$ No Persistence

10 Male $20-29 \quad 0 \quad 0 \quad$ SSI $\quad$ IPM, CST, $\quad$ No $53 \quad$ Yes $\quad$ No Undetermined CES

11 Male 80-89 $4 \quad 2 \quad$ CLABSI $\quad$ MEM, $\quad$ Yes $17 \quad$ Yes $\quad$ Yes Undetermined CST, TIG

12 Male 40-49 $3 \quad 2 \quad$ VAP $\quad$ MEM, $\quad$ No $28 \quad$ No $\quad$ No Persistence CST, CES,

DOX

13 Male $30-39 \quad 0 \quad 2 \quad$ VAP $\quad$ MEM, CST $\quad$ No $21 \quad$ Yes $\quad$ No Undetermined




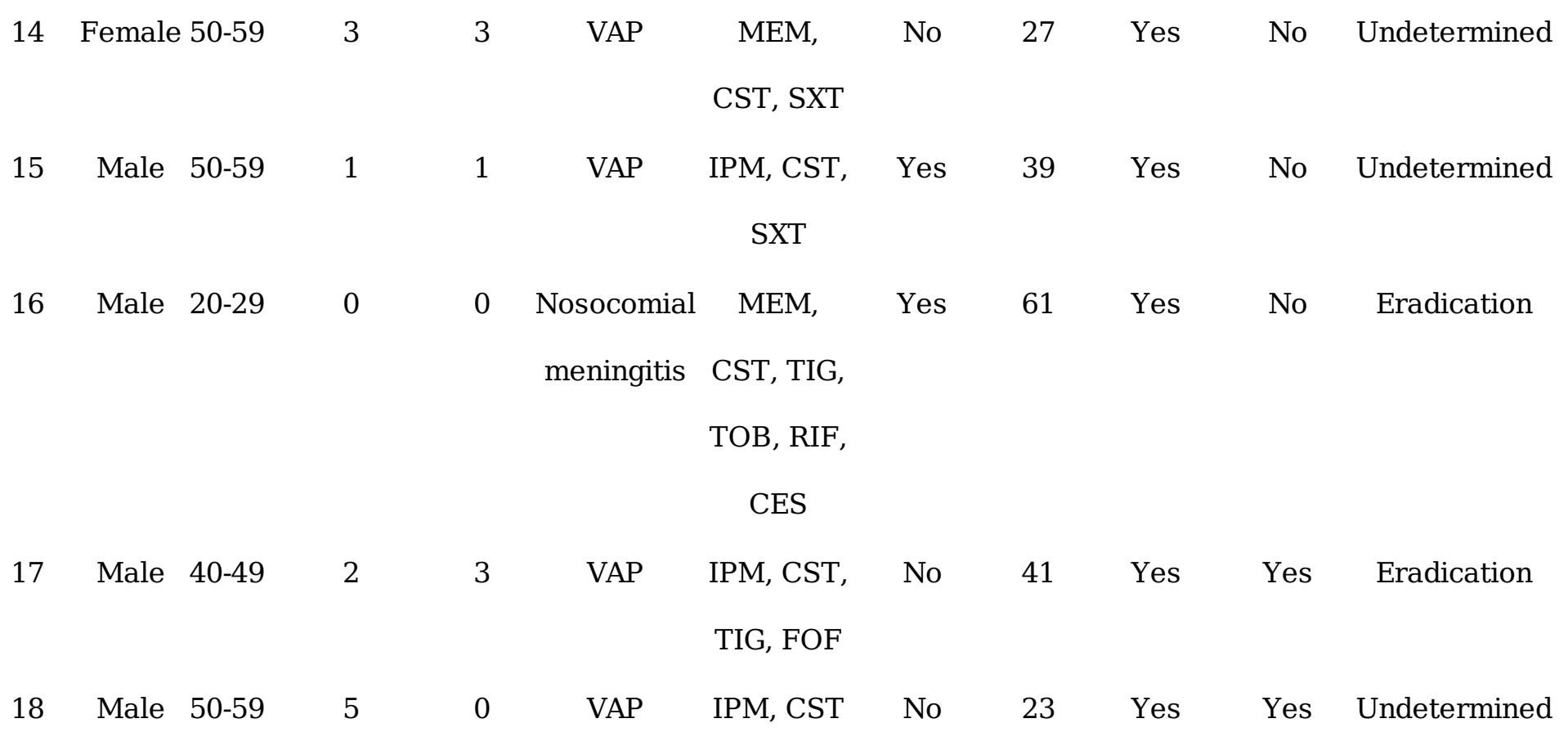

\begin{abstract}
Abbreviations: qSOFA, quick Sepsis Related Organ Failure Assessment; TZP: Piperacillin tazobactam; VAN, Vancomycin; EVD, Extraventricular drain; CST, colistin; HAP, hospitalacquired pneumonia; ICU, intensive care unit; MEM, meropenem; RIF, rifampin; TIG, tigecycline; VAP, ventilator-associated pneumonia, MIN, Minocycline; CES, Cefoperazonesulbactam, IV, Intravenous; IT, Intra thecal; SXT, Trimethoprin sulphamethoxazole; IPM, Imipenem; DOX, Doxycycline; FOF, Fosfomycin, AMK, Amikacin, TOB, Tobramycin; CSF, Cerebrospinal fluid, CLABSI, Central line associated Bloodstream Infection; SSI, Skin \&Soft tissue Infection; CVC, Central venous Catheter
\end{abstract}

Table 2: Comparison of clinical features between those who recovered versus those who died 
Died $(n=8)$ Recovered

$$
(\mathrm{n}=10)
$$

Gender (M:F)

$6: 2$

9:1

Age (mean years)

62.25

41.5

Charlson's Index $<3$

2

7

$>=3$

6

3

qSOFA score

$<2$

2

4

$$
>=2
$$

6

6

Source of Infection

Respiratory tract

$\begin{array}{ll}6 & 7\end{array}$

Wound infection

0

2

CLABSI

1

0

CNS infection

1

1

Presence of devices

CVP line

5

8

ET tube

6

8

Sensitive antibiotic used

5

4

Antibiotic duration (mean days) 16

22

Length of stay (mean days)

28

31

ICU admission

7

7

\section{Figures}




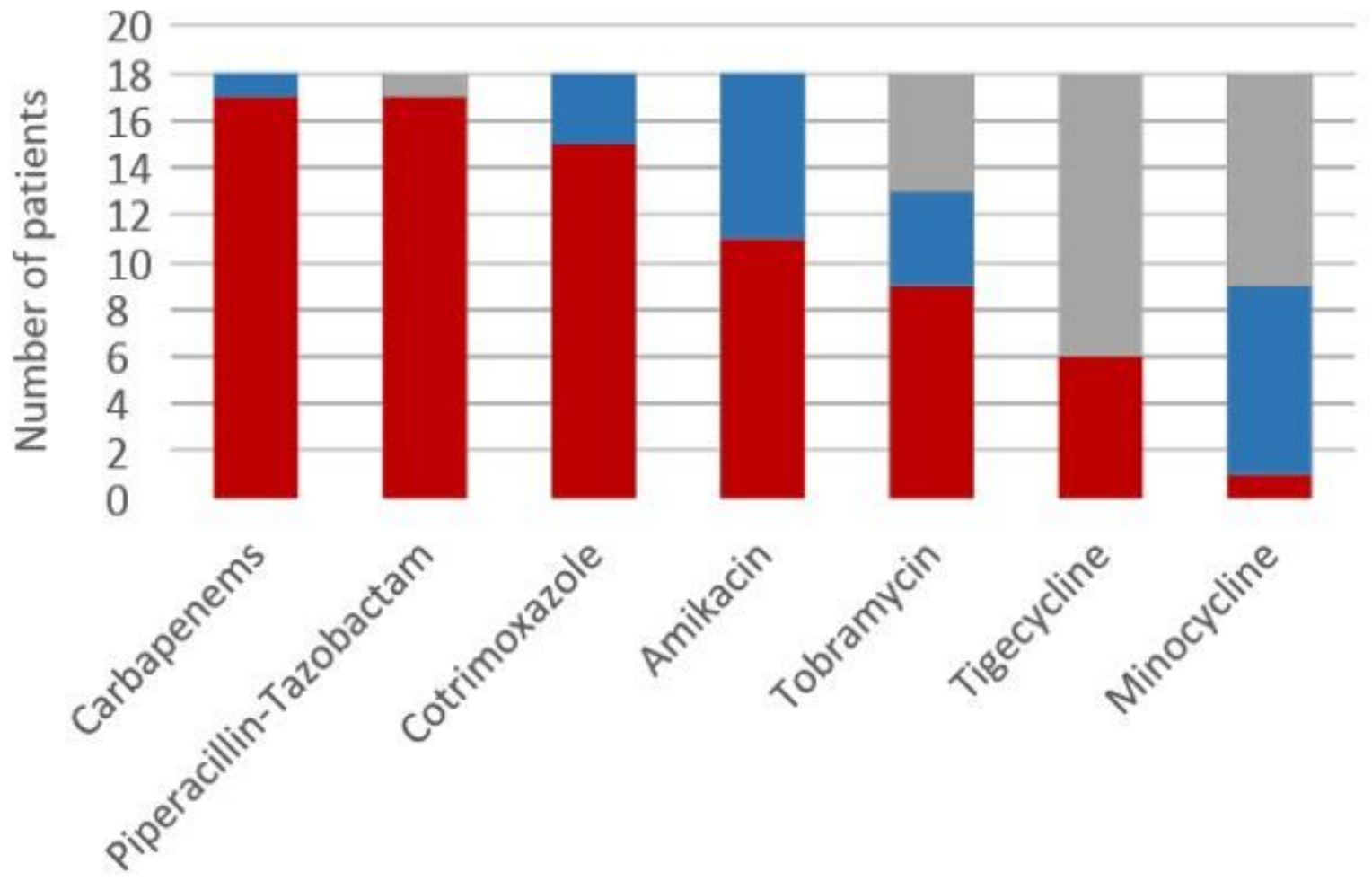

- Resistant $\mathbf{a}$ Sensitive $\mathbf{n}$ Not Checked

\section{Figure 1}

Antimicrobial susceptibility pattern of Acinetobacter species 